\title{
Chilueno o arauco, idioma de los changos del norte de Chile, dialecto mapuche septentrional
}

Queremos presentar aquí una información inédita que nos parece importante en relación con la lingüística indígena de una región de América que nos es querida, recordando los años que hemos vivido allí, cuando enseñábamos en la Universidad del Norte, en Antofagasta.

En efecto, hemos descubierto un antiguo documento que hasta ahora parece haber escapado completamente a la atención de los bibliógrafos. Se trata de: "Le désert d'Atacama et Caracoles (Amérique du Sud), M. l'Ingénieur A. Bresson, 1870-1874. Texto et dessins inédits". ${ }^{2}$

Este título no se menciona en el Handbook of South American Indians, ni en América Indígena de Pericot y García, ni en Loukotka. Parece que este relato de viaje pasó inadvertido por los primeros americanistas tales como Chamberlain y Rivet, a quienes no hubiera dejado de interesar de una manera considerable. No hay duda de que si estos primeros autores hubiesen tenido conocimiento del relato del ingeniero Bresson, esto nos hubiera ahorrado una de esas extravagancias clasificatorias que desfiguran tan enfadosamente el panorama de la lingüística sudamericana.

La extravagancia en este caso consiste en hacer de los changos de la costa norte de Chile una población de lengua uru, hipótesis todavía acogida en la última clasificación de Loukotka (1968). Sin embargo, el análisis del problema hecho por Mason y Bird en el Handbook of South American Indians no dejaba duda alguna sobre la validez de este emparentamiento uru-chango, ni de cualquier otro parentesco que se podría proponer del chango con el atacameño, el chono o el alacaluf.

1 Departamento de Lingüística, Universidad Nacional Mayor de San Marcos, Lima, PERU.

2 En: Le tour de monde. Nouveau Journal des Voyages, T XXIX (750-751: 321-352 (1875).
En efecto, Mason subrayaba con pertinencia: "No disponemos más que de informaciones insuficientes para la clasificación de chango, lengua extinta de pescadores de la costa chilena. Si consideramos éstos como posibles descendientes de una población arcaica, no sería inverosímil suponer que hablaban un idioma independiente. Pero esta posibilidad es demasiado débil para justificar el establecimiento de una familia separada para ellos solos, como lo hizo Chamberlain en 1913." El mismo Chamberlain fue quien escribía en 1910: "No tenemos ni vocabulario ni textos en lengua chango, pero su calidad como familia lingüística independiente ha sido afirmada por los indígenas mismos.’(!)

Ya en el momento en que Chamberlain firmaba estas líneas habían transcurrido 35 años desde que el ingeniero Bresson publicó su relato de viaje, iy éste incluía una lista de palabras de la lengua chango! En las páginas 326-327 se encuentran, dando cuenta de observaciones efectuadas en 1870 , una breve pero interesante descripción etnográfica.

"En Paposo, hemos tenido la oportunidad de observar indios de la tribu de los changos, curiosos restos de una población primitiva. Los changos son todos perscadores; del mar extraen el alimento que no les puede dar el desierto, que comienza desde la playa hasta perderse de vista. Para establecer su pueblo escogen los alrededores de una aguada que muchas veces les da una agua no muy buena, pero se las arreglan. Su habitación es de una construcción muy simple: fijan en la arena cuatro costillas de ballena -éstas abundan en las playas- y rellenan los intervalos con piel de foca o con trapos. En el interior no se encuentra ni cama ni asiento ni mesa. El único mueble es una odre hecha de un estómago de foca y que sirve para el transporte y la conservación de la provisión de agua dulce que traen de la aguada vecina."

"Para pescar usan unas embarcaciones muy extrañas, llamadas balsas. Estas están compuestas por dos cilindros de cuero de foca inflados y dispuestos 
paralelamente. Las extremidades terminan en puntas un poco levantadas. Las dos partes del esquife son unidas por pequeños travesaños sobre los cuales se extiende otra piel de foca. Es sobre esta especie de puente que se sientan o acurrucan los changos. Mueven sus embarcaciones por medio de remos de madera y cuentan mucho con su habilidad para aventurarse en viajes muy lejanos". En la p. 328 hay un dibujo de T. Weber (según una fotografía) que representa una balsa de los changos.

El autor, después de haber subrayado que "los changos son consumidores muy apasionados de hojas de coca" y después de haber descrito ampliamente el consumo y los efectos de este excitante, sigue:

\section{Español}

Cabeza

Cabello

Nariz

Ojo

Boca

Orejas

Brazo

Pies

Hombre

Mujer

Niño

Niña

Uno

Dos

Tres

Cuatro

Cinco

Seis

Siete

Ocho

Nueve

Diez

Cien

Arauco
lonco
thopel
yu
gue
un
pilun
riun
namun
che
domo
hueche
malguen
quiné
epu
quúla
meli
quechu
cayu
relge
pura
ailla
marí
pataca

"Recogí algunas palabras del idioma de esos changos, el chilueno o arauco; eso no sin pena, porque esa lengua primitiva está desapareciendo cada vez más; la raza de los changos no cuenta ahora más que con 250 hasta 300 individuos, y casi todos han abandonado su idioma nacional por el español.

"He aquí el fragmento de vocabulario. Relaciono las palabras del arauco con las palabras correspondientes, pero completamente distintas de la lengua de los atacameños, otra tribu más al norte, que también tiende a desaparecer.

"Son éstos documentos auténticos, es preciso asegurar su conservación:

Atacameño
hlacse
musa
sepe
ikhcpe
khaipe
aïke
soke
khoche
sima
likan
sima pauna
likan pauna
sema
poya
palama
chalpa
mutsma
michelo
ch'hoya
ch'olo
teker
such
hara

A continuación, presentamos la confrontación de la lista de palabras chilueno-arauco de Bresson con el Diccionario comentado mapuche-español (araucano - pehuenche - pampa - picunche - rancülche - huilliche), de Esteban Erize (Cuadernos del Sur, Buenos Aires, 1960): 


\section{Bresson (chilueno-arauco)}

$\begin{array}{ll}\text { Cabeza } & \text { lonco } \\ \text { Cabello } & \text { thopel } \\ \text { Nariz } & \text { yu } \\ \text { Ojos } & \text { gue } \\ \text { Boca } & \text { un } \\ \text { Orejas } & \text { pilun } \\ \text { Brazo } & \text { riún } \\ \text { Pies } & \text { namun } \\ \text { Hombre } & \text { che } \\ \text { Mujer } & \text { domo } \\ \text { Niño } & \text { hueche } \\ \text { Niña } & \text { malguen } \\ \text { Uno } & \text { quiné } \\ \text { Dos } & \text { epu } \\ \text { Tres } & \text { quúla } \\ \text { Cuatro } & \text { meli } \\ \text { Cinco } & \text { quechu } \\ \text { Seis } & \text { cayu } \\ \text { Siete } & \text { relge } \\ \text { Ocho } & \text { pura } \\ \text { Nueve } & \text { ailla } \\ \text { Diez } & \text { mari } \\ \text { Cien } & \text { pataca }\end{array}$

Sin lugar a dudas, el vocabulario recogido por el ingeniero Bresson en 1870 de la boca de los changos de Paposo es indiscutiblemente mapuche. Por otra parte, no tenemos ningún motivo para dudar de la buena fe del ingeniero Bresson. Su descripción etnográfica no deja ninguna duda de que fue a los changos a quienes interrogó y no a algún mapuche trasplantado casualmente a Paposo. No siendo Bresson ni antropólogo ni lingüista, y estando él notablemente más interesado en minas y en ferrocarriles, no se le puede sospechar de distorsionar los hechos con miras a confirmar una teoría preconcebida. Además está a la vista que no se dio cuenta el mismo Bresson, que la lengua de los changos era la misma que la que hablaban otros indígenas más al sur.

Su única preocupación era demostrar la falta aún evidente de parentesco lingüístico de los indios changos con los atacameños, que Bresson conoció también muy bien, cuando hizo sus prospecciones en el interior del desierto.

Hasta ahora, las estimaciones más generosas extendieron el área de repartición de las lenguas mapuche hasta Copiapó ( $27^{\circ} 20^{\prime}$ Lat. S). Sobre la base de este documento olvidado, estaríamos obligados a

\section{Erize \\ (mapuche común)}

$\begin{array}{ll}\text { lonco } & \text { cabeza } \\ \text { topel } & \text { cabello } \\ \text { yüu } & \text { nariz } \\ \text { ngue } & \text { ojos } \\ \text { uún } & \text { boca } \\ \text { pilun } & \text { orejas } \\ \text { riuncúg } & \text { biceps } \\ \text { namun } & \text { pies } \\ \text { che } & \text { ser humano } \\ \text { domo } & \text { mujer } \\ \text { hueche } & \text { hombre joven }-18 / 25 \text { años } \\ \text { malguen } & \text { virgen } \\ \text { quiñe } & \text { uno } \\ \text { epu } & \text { dos } \\ \text { cula } & \text { tres } \\ \text { meli } & \text { cuatro } \\ \text { quechu } & \text { cinco } \\ \text { cayu } & \text { seis } \\ \text { relgue } & \text { siete } \\ \text { pura } & \text { ocho } \\ \text { ailla } & \text { nueve } \\ \text { marí } & \text { diez } \\ \text { pataca } & \text { cien }\end{array}$

prolongar este dominio hacia el norte, por lo menos hasta Paposo $\left(25^{\circ}\right.$ Lat. S). Parece que había changos mucho más al norte, hasta Cobija, quizás hasta las cercanías de Anca. Pero Bresson, quien tenía que conocer muy bien la costa entre Cobija y Mejillones, ya que durante años tuvo allí su residencia, en ningún momento menciona a los changos en esta región. Como los había conocido en Paposo, si les hubiese encontrado también en esta zona, no hubiera dejado de darnos cuenta de ello.

De todos modos, tomar en consideración la lista de palabras chilueno-arauco de Bresson hace definitivamente obsoletas las clasificaciones acrobáticas para reagrupar la lengua de los changos con el uru, el chono o el alacaluf, y se vuelve a demostrar la inutilidad de suponer una lengua chango independiente. ${ }^{3}$

\footnotetext{
3 Nota de Lautaro Núñez: Philippi (1860) puntualiza la presencia de lengua araucana en la costa del Desierto de Atacama, pero no fundamentó su observación. El Dr. D’Ans entrega ahora una información que abre nuevas perspectivas para la etnolingüística regional. El problema parece ser complejo. Dagnino (1909) presenta documentos que demuestran tempranos traslados de esclavos araucanos a Anca (1627). Esperamos nuevas indagaciones de los especialistas.
} 


\section{APENDICE}

IV

De Mejillones a Valparaíso. Paposo. Les changos. Les balsas. Le coca. Les atacameños. Oébris de leur idiome. Les aymaras. La montera et le topo.

De Mejillones a Valparaíso, notre steamer fit de très-nombreuses escales le long des côtes chiliennes; nous visitions parfois trois ports dans la même jornée, mais ils étaient tous aussi monotones et aussi sales d'aspect les unes que les autres. Une usine pour traiter les minerais de cuivre du voisinage, des masures pour les mineurs, des distilleries d'eau de mer, voilà ce qui se représente sans cesse.

A Paposo, nous eûmes l'occasion d'observer des indiens de la tribu des changos, débris curieux de la population primitive. Les changos sont tous pêcheurs; ils demandent à la mer l'alimentation que ne peut leur donner le désert, qui commence dès la plage pour se prolonger à perte de vue. Ils choisissent, pour établir leur village, les environs l'une aguade, dont l'eau est souvent fort médiocre, mais ils s'en contentent. Leur habitation est d'une construction très simple: ils plantent dans le sable quatre côtes de baleine -les plages en sont jonchées- puis ils garnissent les intervalles avec des peaux de phoque ou de vieilles toles. A l'intérieur, il ne faut chercher ni lit, ni chaises, ni table: le seul meuble est une outre, formée d'un estomac de loup marin, et qui sert au transport et à la conservation de la provision d'eau douce qu'on tire de l'aguade voisine.

\section{Le Desert d'Atacama et Caracoles}

Pour pêcher, ils se servent d'embarcations très étranges, nommées balsas; elles se composent de deux cylindres en cuir de phoque, gonflés d'air, et placés parallêlement. Les extrémités sont terminées en pointe un peu relevée, et les deux parties de l'esquif sont réunies par de petites traverses sur les quelles on étend une autre peau de loup marin; c'est sur cette espèce de pont que sont assis ou accroupis les changos; ils mettent leurs embarcations en mouvement à l'aide de pagaies en bois, et ils se fient assez à leur adresse pour s'aventurer dans des voyages fort lointains.
Les changos sont au nombre des amateurs les plus passionnés des feuilles de coca; ils en mâchent continuellement. Plusieurs d'entre eux vont porter, dans les villes de l'intérieur, du poisson sec et se procurent en échange cette denrée si précieuse a leurs yeux. Elle a pour eux une grande valeur, car elle possède, dit-on, le pouvoir de soutenir les forces en l'absence de toute alimentation; le système nerveux reçoit par la mastication de ces feuilles une excitation qui empêche l'épuisement. C'est grâce au coca, paraît-il, que les indiens, les indiennes, les soldats, les muletiers, font des marches prolongées sans vivres, trottinant sans s'arrêter dans des sables brûlants et mouvants, et toujours contents, pourvu qu'ils aient dans la bouche un reste de leur cher coca.

L'usage de cette mastication remonte au temps des Incas. La feuille est produite par l'arbrisseau nommé par Ant.-Laur. de Jussieu Erythroxylon coca. Le Bolivien porte sa provision dans un petit sac appelé chuspa; lorqu'il veut faire sa chique, qu'il renouvelle à intervalles égaus, il prend, une à une, les feuilles desséchées dout il a besoin, les étale lentement sur la paume de sa main gauche, où il les mouille avec sa langue; au moyen d'une baguette, il y met une petite quantité de chaux ou de pâte alcaline nommée llipta (formée des cendres du quinoa ou du cactus cierge), et roulant le tout en forme de boule, il l'introduit dans le coin de sa bouche.

Le mélange d'un alcali avec la feuille de coca est destiné à neutraliser un principe acide qu'elle contient, en même temps qu'il favorise la production de la salive qui doit la dissoudre.

La feuille de l'Erythroxylon ressemble assez à celle du thé, mais elle n'a pas de dentelures; son odeur est également analogue à celle du thé; en infusion, elle a une amertume sui generis. Prise comme tisane, elle donne un peu d'exitation et d'insommie. Elle ne peut être mâchée sans conséquences fâcheuses par l'Européen qui n'a pas cette habitude dès l'enfance.

J'ai pu recueillir quelques mots de la langue de ces changos, le chilueno ou arauco; ce n'est pas sans peine, car cet idiome primitif disparaît de plus en plus; la race des changos ne compte guère maintenant que deux cent cinquante à trois cents individus, et 
presque tous ont abandonné leur langue nationale pour l'espagnol.

Voici ce fragment de vocabulaire; j'en rapproche les mots correspondants et tout à fait distincts de la langue des atacameños, autre tribu, plus au nord, qui tend aussi à disparaitre. Ce sont là des documents authentiques dont il faut se hàter d'assurer la conservation:

\begin{tabular}{|c|c|c|}
\hline Français & Arauco & Atacameño \\
\hline Tête................... & & hlacse \\
\hline Cheveux ........ & thopel. & musa \\
\hline Nez ................. & yu.................... & sepe \\
\hline Yeux .................... & gue ................... & ikhepe \\
\hline Bouche .......... & un........................ & khaipe \\
\hline Oreilles.......... & pilun ............... & â̂ke \\
\hline Bras .................. & riun .................. & soke \\
\hline Pieds ................ & namun............. & khoche \\
\hline Homme.......... & che ................. & sima \\
\hline Femme........... & domo .............. & likan \\
\hline Petit garçon .. & hueche ........... & sima pauna \\
\hline Petite fille..... & malguen......... & likan pauna \\
\hline Un ...................... & quiné.............. & sema \\
\hline Deux ................. & epu................. & poya \\
\hline Trois ............... & quúla.............. & palama \\
\hline Quatre............. & meli ................. & chalpa \\
\hline Cinq................ & quechu ........... & mutsma \\
\hline Six ...................... & cayu .................. & michelo \\
\hline Sept .................... & relge.............. & ch'hoya \\
\hline
\end{tabular}

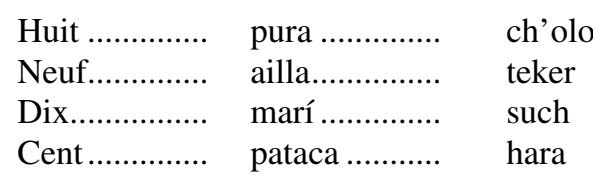

On voit combien ces deux idiomes, malgré leur voisinage, sont différents. L'un est bien plus dur que l'autre.

A côté des changos et des atacameños, on trouve en Bolivie les aymaras, qui sont la race véritable indigène et nationale. Il faut avouer qu'elle n'est pas belle. Je n'ai jamais rencontré un visage qui fût seulement passable. Pour comble de malheur, le costume adopté par les femmes aymaras est aussi disgracieux que possible. La pièce la plus caractéristique est le chapeau appelé montera, dont l'aspect se modifie beaucoup, selon son état de vétusté plus ou moins avancé. Sa forme est celle d'une énorme corolle dont le limbe aurait environ deux pieds. Le reste du costume consiste en un nombre indéterminé de jupes noires ou bleu foncé, qui sont plus ou moins abondantes selon la fortune et l'âge; à ces jupons s'ajoute une camisole de même étoffe recouverte d'un gros fichu de serge oblong; les bouts de ce fichu, ramenés sur la poitrine, sont fixés au moyen d'une énorme épingle, appelée topo, dont la tête a tout à fait la forme d'une cuiller. Hommes et femmes tressent leurs cheveux en un grand nombre de petites nattes qui pendent sur le cou, ou sont réunis en queue avec un cordon. 


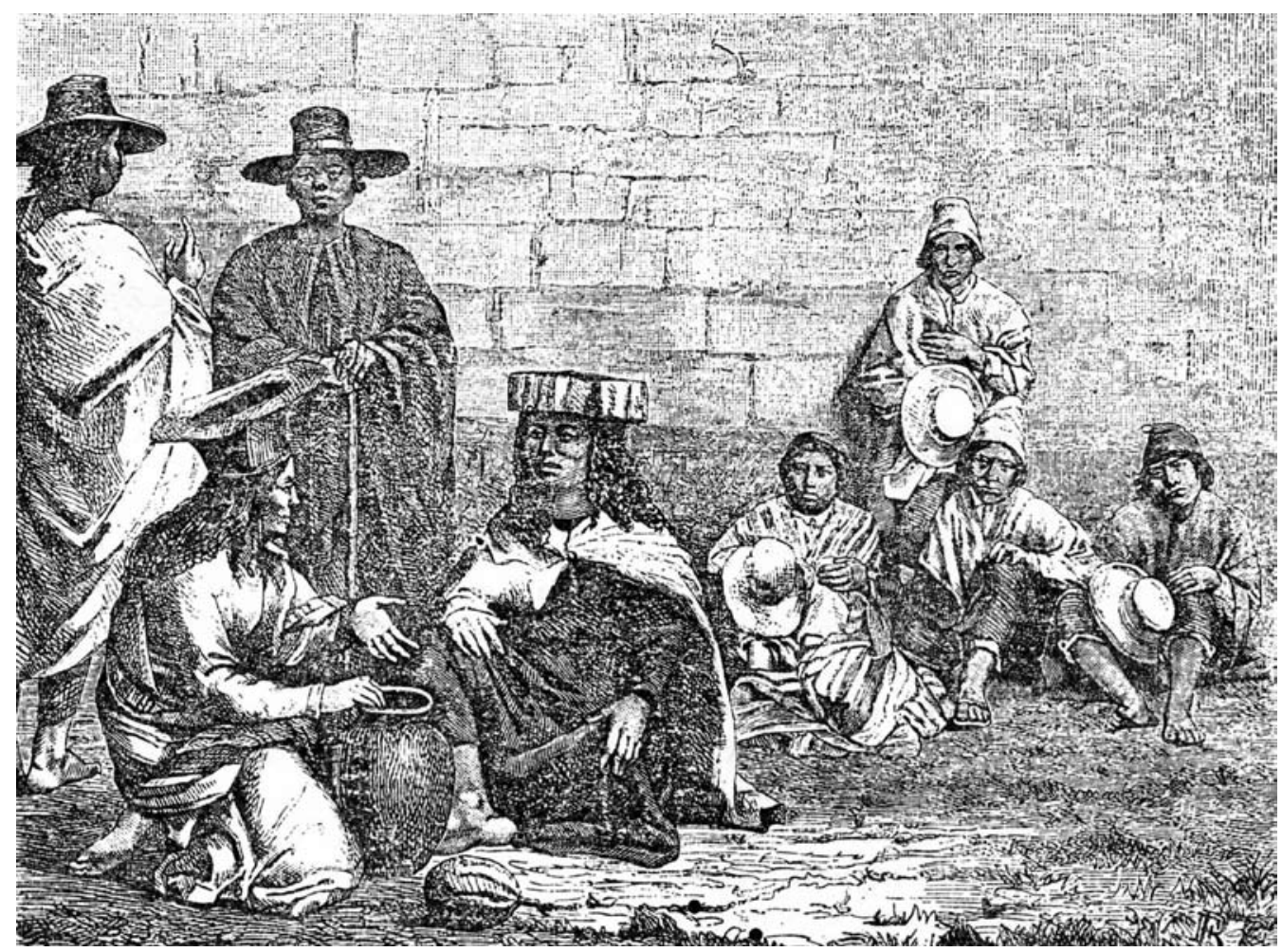

Indiens atacameños et aymara. Dessin de Bassot, d'après une photographie. Nota del autor: Atacameño en el centro.

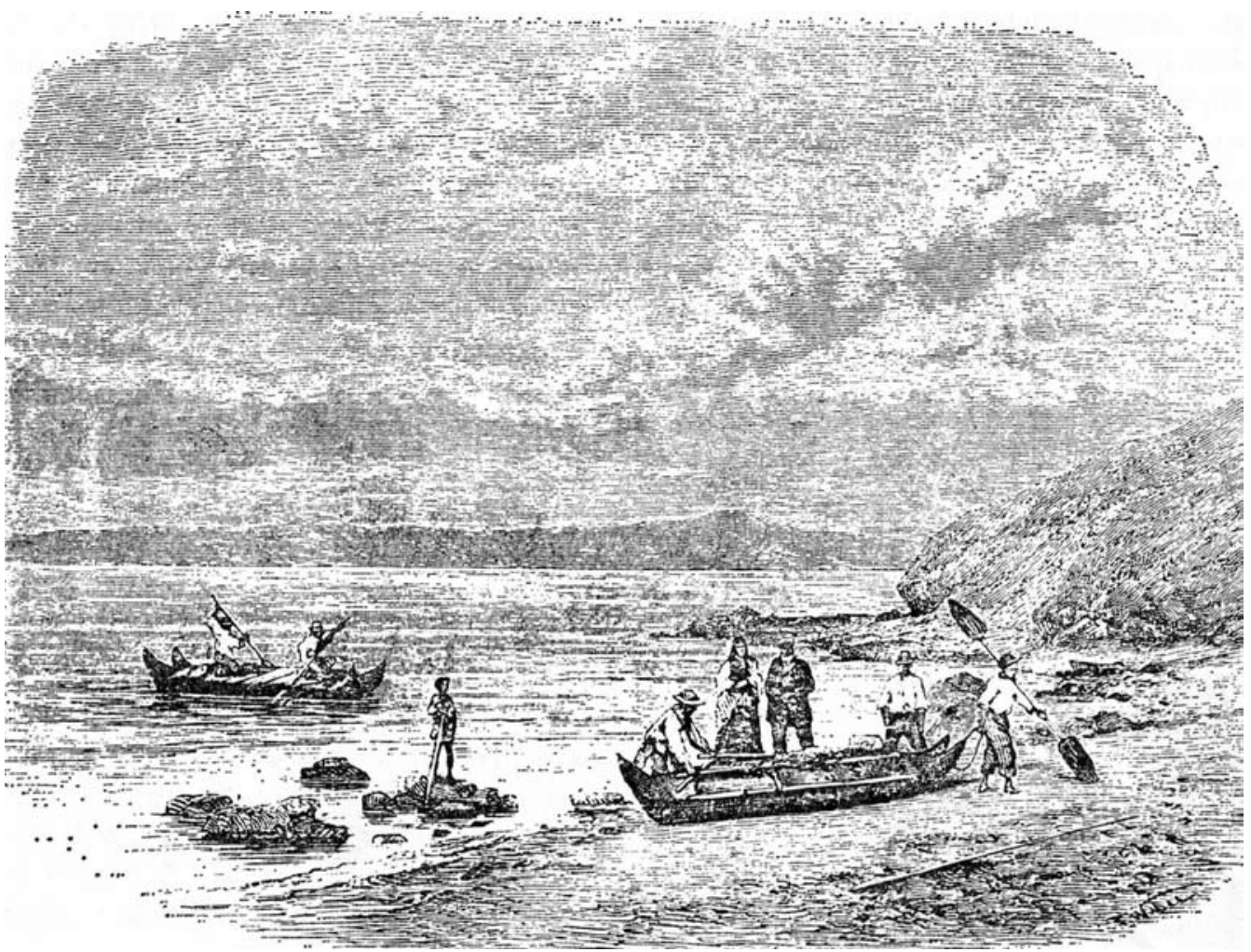

Indiens changos: Barques balsas. Dessin de tn. Weler, d'après une photographie. 